\title{
Patients' Involvement in Decision Making at End of Life-A Systematic Review of Literature
}

\author{
Elham H. Othman'1 (), Inaam Khalaf', Ruqayya Zeilani² \\ ${ }^{1}$ King Hussein Cancer Center, Amman, Jordan, School of Nursing, The University of Jordan, Amman, Jordan \\ ${ }^{2}$ School of Nursing, The University of Jordan, Amman, Jordan \\ Email: eothman@khcc.jo,elham.othman@ymail.com,khalafd@ju.edu.jo,khalaf12@yahoo.com,zeilani.r@gmail.com, \\ zeilani.r@gmail.com
}

How to cite this paper: Othman, E.H., Khalaf, I. and Zeilani, R. (2019) Patients' Involvement in Decision Making at End of Life-A Systematic Review of Literature. Open Journal of Nursing, 9, 1106-1121. https://doi.org/10.4236/ojn.2019.910081

Received: September 30, 2019

Accepted: October 28, 2019

Published: October 31, 2019

Copyright $\odot 2019$ by author(s) and Scientific Research Publishing Inc. This work is licensed under the Creative Commons Attribution International License (CC BY 4.0).

http://creativecommons.org/licenses/by/4.0/

\begin{abstract}
Background: involving patient in end of life decision is important to understand their wishes and preference, which will help health care providers in improving the quality of dying and minimizing suffering. Aim: the aim of this review was to provide a detailed examination of the available literature related to patients' involvement in decision making at end of life. Design: a systematic review following the PRISMA protocol was used, the review protocol was registered on PROSPERO: CRD42019128556. Data sources: we conducted a literature search in two electronic databases "CINAHL and Medline" during March-April 2019. The retrieved articles were included if they were: research reports or literature review; examined patient involvement in end of life discussions; full text publications, written in English and published from 2000-2019. Results: a total of (22) articles were included in the review; there was diversity in the purposes and design approach of the retrieved studies. The available literature explored patient's involvement at end of life decision making through; describing current practices; understanding perspectives of end of life discussions; investigating the impact and identifying the barriers and facilitators of patients' involvement in end of life discussions. Conclusion: involvement in end of life discussions improved the recognition of patients' wishes, improved death experience, and decreased posttraumatic stress, depression, and anxiety among family members. Despite the documented benefits, some barriers against patient's involvement in EOL decisions were recognized; lack of awareness; lack of education, training and experience; concerns about ethical and legal issues; and personal preferences of doctors or nurses were among the most commonly identified barriers.
\end{abstract}

\section{Keywords}

Advance Directives, Advance Care Planning, Decision Making at End of Life, 
Patients' Involvement, Patients' Preferences, Systematic Review

\section{Introduction}

\subsection{Background}

Being hospitalized or having a hospitalized family member is overwhelming and a stressful experience, especially if the illness is terminal and death is imminent. Patients, their family caregivers and health care providers (HCPs) are obligated to make crucial decisions challenged by the sensitive circumstances surrounded by end of life (EOL) which increase the difficulty of making them [1].

Patients differ in their preference for participation in treatment decisions at EOL [2]. The goal of involving patient in EOL decision making is to understand the patient's values and treatment preferences [3], promote autonomy and empower patients and their caregivers [4], as well as, recognize the patients' wishes [5], improve EOL care from the perspective of the patient and diminish the likelihood of stress, anxiety, and depression in surviving relatives [6].

Even though the HCPs recognize the importance of ongoing communication and providing adequate information about the patient's condition; reports indicate that the majority of family members of in-hospital deceased patients were dissatisfied with HCPs' communication regarding EOL decisions, and they clearly verbalized their preferences to have more communication regarding their patient's condition [7]. It is necessary to evaluate the available evidence in order to enhance patients' involvement in EOL decision making.

\subsection{Review Aim}

The aim of this review is to provide a comprehensive understanding of patients' involvement in decision making at EOL. More specifically, the review aims to answer two research questions: what is the level of patients' involvement in the EOL decision making and what are the patients' preferences regarding participation in EOL decisions?

\subsection{Objectives}

1) Systematically identify, analyze and describe studies that evaluated patients' involvement in decision making at EOL.

2) Evaluate the nature and strength of evidence for patients' involvement in EOL decision making and patients' preferences regarding participation in EOL decisions.

\subsection{Outline of the Content}

This review was written following the Preferred Reporting Items for Systematic 
Reviews and Meta Analyses (PRISMA) protocol for systematic reviews [8]. The main sections of this manuscript are: introduction, methods, results and discussion. The first section is the introduction which includes a brief background and explanation of the review aim and objectives. The methods section is composed of systematic steps that are followed to search and retrieve the eligible studies, extract data in a rigorous way and examine the quality of evidence derived from the included studies. The last section is the discussion which includes summary of evidence and conclusion.

\section{Methods}

\subsection{Protocol and Registration}

The protocol for this review was registered on the PROSPERO and is available at the PROSPERO registry at [http://www.crd.york.ac.uk/PROSPERO/display record.php?ID=CRD42019128 556].

\subsection{Eligibility Criteria}

We referred to the PICOT framework to guide our search; including population of interest (P), issue of interest (I), comparison of interest (C), outcome of interest $(\mathrm{O})$ and timeframe $(\mathrm{T})$ [9]. Our search focused on research studies published since 2000, and addressed patient participation in decisions at EOL.

The retrieved articles were included if they were: 1) research reports or literature review; 2) examined patient involvement in EOL discussions; 3) involved adult patients; 4) full text publications, written in English and 5) published from 2000-2019.

\subsection{Information Sources}

Two nurse researchers (EO, IK) independently searched CINAHL and Medline databases to find eligible publications. The initial search of the literature was performed between March $3^{\text {rd }}, 2019$ and April $20^{\text {th }}, 2019$.

\subsection{Search}

Research articles and systematic reviews of EOL discussions were identified through the search. Search terms included EOL discussions, decision making at EOL and patient's involvement. Additionally, the reference list of the articles was searched to identify possible publications not retrieved electronically. Mendeley software was used to find duplicates and facilitate citation.

\subsection{Study Selection}

Similarly, two researchers (EO, RZ), screened titles and abstracts. Then, full text of these potentially eligible studies was assessed for eligibility. Any disagreement over the eligibility of a particular study was resolved by a third reviewer (IK). 


\subsection{Data Collection Process}

Data were extracted by two reviewers (EO, RZ) using a developed data extraction sheet.

\subsection{Data Items}

The reviewers read the retrieved studies in-depth and summarized it in two tables: 1) studies characteristics (target population, sample, settings, research design, and country), and 2) studies' findings

\subsection{Risk of Bias in Individual Studies}

Two reviewers (EO, IK) assessed the risk of bias in all studies. The Quality Assessment Tool (QAT) checklist was used to evaluate the quantitative studies [10], while the Critical Appraisals Skills Programme (CASP) Checklists were used to evaluate the qualitative studies and reviews [11] [12]. Disagreements were resolved by a third reviewer (RZ).

\subsection{Synthesis of Results}

Data were synthesized and analyzed by two reviewers (EO, IK), data were included only if both reviewers agreed on. The main findings are presented in tables and in a narrative synthesis.

\section{Results}

\subsection{Study Selection}

Initial database searches retrieved 1203 articles; after removing duplicates, the titles and abstracts of 491 articles were screened. One hundred twenty studies were selected for full text screening, 22 of them were found eligible and included in the review. The search and selection process are shown in the PRISMA flowchart (Figure 1) [8].

\subsection{Study Characteristics}

A total of (22) final articles published between 2000 and 2019, were included in the review. Most of the selected studies were review papers $(n=8)$ [13]-[20], followed by qualitative research studies $(n=6)$ [1] [2] [4] [21] [22] [23], nonexperimental studies $(n=6)$ [5] [24] [25] [26] [27] [28], and lastly two clinical trials [6] [29].

The articles included different population and settings; the settings involved seven hospitals [1] [2] [6] [13] [19] [24] [28], four outpatients [5] [25] [26] [27], four nursing home settings [4] [18] [22] [23], one hospice agency [21], and three multi sites [14] [15] [29]. Furthermore, the selected studies used different sources of data; the majority targeted patients $(n=11)$ [2] [6] [15] [21] [23]-[29], patients and care provider $(n=6)$ [4] [5] [14] [18] [19] [22], and two studies collected data from healthcare providers (HCPs) [1] [20]. The characteristics of selected articles are summarized in (Table 1). 


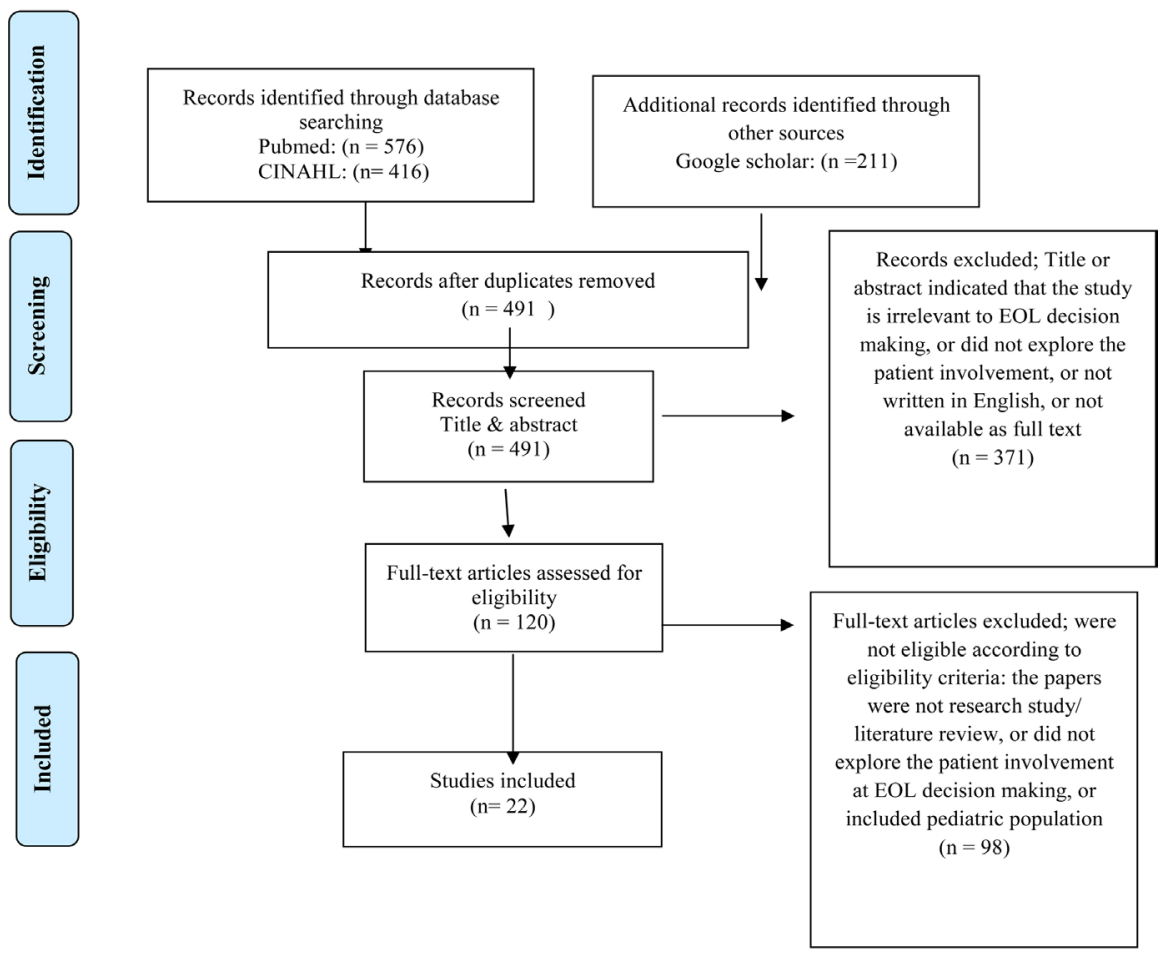

Figure 1. PRISMA flowchart. PRISMA: Preferred Reporting Items for Systematic Reviews and Meta Analyses.

Table 1. Characteristics of literature included in review $(\mathrm{N}=22)$.

\begin{tabular}{|c|c|c|}
\hline & $\mathrm{N}(\%)$ & References (No.) \\
\hline \multicolumn{3}{|c|}{ Target Population } \\
\hline General & $7(32)$ & [6] [14] [15] [17] [22] [25] [26] \\
\hline Critical care patients & $4(18)$ & [1] [13] [19] [24] \\
\hline Palliative care patients & $2(9)$ & {$[20][21]$} \\
\hline $\begin{array}{l}\text { Patient with specific situa- } \\
\text { tions }\end{array}$ & $6(27)$ & $\begin{array}{l}\text { Cancer: [2] [5] [27] Cardiac: [28]; COPD: [29]; } \\
\text { Dementia: [16] }\end{array}$ \\
\hline Nursing homes residents & $3(14)$ & [4] [18] [23] \\
\hline \multicolumn{3}{|c|}{ Sample } \\
\hline Patients & $11(50)$ & [2] [6] [15] [21] [23]-[29] \\
\hline HCPs & 2(9) & [1] [20] \\
\hline Patients and providers & $6(27)$ & [4] [5] [14] [18] [19] [22] \\
\hline \multicolumn{3}{|c|}{ Settings } \\
\hline Inpatients & $7(32)$ & [1] [2] [6] [13] [19] [24] [28] \\
\hline Outpatients & $4(18)$ & [5] [25] [26] [27] \\
\hline $\begin{array}{l}\text { Nursing home } \\
\text { settings/hospice agencies }\end{array}$ & $5(23)$ & [4] [18] [21] [22] [23] \\
\hline Multi-settings & $3(14)$ & [14] [15] [29] \\
\hline
\end{tabular}




\section{Continued}

\begin{tabular}{lll}
\hline & \multicolumn{2}{c}{ Study Design } \\
\hline Experimental & $2(9)$ & {$[6][29]$} \\
Non-experimental & $6(27)$ & {$[5][24][25][26][27][28]$} \\
Qualitative & $6(27)$ & {$[1][2][4][21][22][23]$} \\
Review & $8(36)$ & {$[13]-[20]$} \\
\hline & \multicolumn{2}{c}{ Country } \\
\hline Australia & $4(18)$ & {$[1][6][27][29]$} \\
Italy & $1(5)$ & {$[18]$} \\
Netherlands & $2(9)$ & {$[2][15]$} \\
Norway & $2(9)$ & {$[4][22]$} \\
Saudi Arabia & $1(5)$ & {$[24]$} \\
Spain & $1(5)$ & {$[19]$} \\
Taiwan & $1(5)$ & {$[17]$} \\
UK & $2(9)$ & {$[16][20]$} \\
USA & $8(35)$ & {$[5][13][14][21][23][25][26][28]$} \\
\hline
\end{tabular}

\subsection{Risk of Bias within Studies}

Quality of included studies was appraised and reported using a trustworthy checklist. Detailed tables of appraisal are presented in the online supplementary material (risk of bias in individual studies-Tables A1-A3).

The quality of quantitative studies was assessed using the QAT, with reference to the tool dictionary and a research study that examined the tool components [30] [31].

Generally, study reports that used quantitative designs showed an adequate quality rating (supplementary risk of bias in individual studies-Table A1). Six studies out of 8 was rated as fair or good on controlling for the selection bias [6] [24] [26] [27] [28] [29] only two studies used clinical trial design [6], [29] and six studies used a cross sectional observational design [5] [24] [25] [26] [27] [28]. Almost 50\% of the studies addressed the confounder variables [6] [25] [26] [29], five studies reported the reliability and validity of the data collection tools [5] [6] [25] [28] [29], and seven studies described the withdrawal rate [5] [6] [24] [26] [27] [28] [29].

The appraised qualitative studies exhibited a high level of quality on the CASP (qualitative) checklist (supplementary risk of bias in individual studies-Table A2). However, two studies failed to state a clear aim of the research, and most of the studies did not adequately describe the relationship between researcher and participants. Similarly, most of the reviews included failing to mention a specific research question and almost three reviews did not include any assessment of the quality of the included studies (supplementary risk of bias in individual studies-Table A3). 


\subsection{Results of Individual Studies}

A thematic synthesis of the re-search studies revealed three categories. A detailed summary of the studies is provided in the online supplementary material (summary of included studies-Table A4).

\subsubsection{Evaluate the Current Practices of EOL Discussions}

Almost seven studies evaluated the current practices of patient's involvement in EOL decision making; either by 1) describing the current practices, 2) examining the relation of EOL decisions with ethical viewpoint, or 3) exploring the role of patients in EOL decisions.

The studies showed a lack of involving patients in EOL decision making. A qualitative descriptive study reported that most patients did not have the opportunity to discuss their values and preferences for EOL treatment, similarly, relatives stated that staff did not initiate discussions with them [22]. Actually, few patients discussed the type of care they want to receive with their family, and fewer had discussed this with their doctor [25] [27] [28]. In the same context, few patients documented their wishes in a written documentor appointed a surrogate decision maker [14] [27]. Nevertheless, it was found that older patients with chronic illnesses, palliative care patients and nursing home residents had a significantly greater completion of any form of $\mathrm{AD}$ [14].

Another major issue was the timing of EOL discussions with patients; inappropriate timing of EOL care discussions was associated with patient and family distress, and discomfort for health care professionals [1]. Generally, there was limited evidence of the timeliness and initiation of EOL discussions [16]. While relatives of patients emphasized on selecting the right timing to initiate discussions [22], it was reported that EOL decisions were made very close to death [24].

Previous studies emphasized that decisions at EOL should combine clinical information about the diagnosis and prognosis with values and beliefs of patients and health care providers [13], most patients have not had the opportunity to discuss their own values and preferences for treatment and care related to EOL [22].

On the other hand, there was a variation in patients' and relatives' involvement; while some family or surrogates were informed and involved in EOL decisions [24], others claimed that family caregivers were not involved or prepared for decision-making [16] [22] and that physicians often make these decisions with little input from others [13].

\subsubsection{Understand Perspectives of EOL Discussions}

The majority of retrieved studies aimed to understand the perspectives toward EOL decision making. Patients' preferences of information disclosure varied from wishing to be fully informed to those who did not want to know everything [22], which means it is important to assess the patient's readiness for an ACP or EOL conversation [15]. Several studies reported patient ambivalence when in- 
volved in ACP [15] [21], uncertainty might be related to prognosis, continued medical treatment, caregiver arrangements, and the circumstances of their dying and death. Patients who support ACP perceived it as a way to control and arrange their lives, decrease their suffering as a result of futile treatments and increase their autonomy, thus enabling their wishes to be respected [17].

A national survey in the USA revealed that the majority of participants prefer to be offered choices and to be asked for their opinions, want to know about their condition and have the option to decide [26]. Additionally, more educated, healthier people, aged up to 45 years were more likely to prefer an active role in decision making, however, people older than 65 years preferred to rely on physician decisions.

Nursing home residents represent a key population in EOL decisions and their perspectives have been examined by several studies [4] [18] [23]. Many elderly and nursing home residents viewed death as a natural part of life [4] [23], they live one day at a time without thinking about death nor about planning their future. While some residents believed that God is in control of life and plans their EOL care [23] others were willing and comfortable talking about EOL care and wanted to make their own decisions [18]. Moreover, it was reported that some older residents had planned the practical issues related to their death (funeral and financial issues) regardless of their preferences of EOL care or decisions [18].

Most nursing home residents reported not having EOL communications with physicians, but they trusted the staff or their family members to be the decision maker regarding EOL care treatments [23]. Relatives, on the other hand, felt insecure about the residents' wishes, experienced decision-making as a burden [4], and preferred shared decision-making with the staff [18]. Participants emphasized on considering cultural differences as the basis for sensitive communication. Likewise, some elders and family members believed that ACP would be initiated gradually in the context of routine care and sensitive to cultural context [23]. Some stated that discussions should start early before the onset of serious health problems or cognitive impairment, residents stressed the importance of both quality of life and a natural death, they did not wish for their lives to be prolonged for no reason, wished for being free of pain and suffer, and having a company at EOL [4] [18].

Results of a systematic review [19] showed that critically ill patients and their relatives have low knowledge about $\mathrm{ADs}$, nevertheless relatives were very interested in receiving information on ADs. Additionally, there are discrepancies between patients' decisions and relatives' decisions, therefore it was suggested that preparing for decision making should start by improving communication between healthcare team, patients and their relatives [19].

Patients from hospice agencies make decisions in the context of the realization that one's life is near its end, and living one day at a time while expressing the importance of having family support for their arrangements [21]. In the same 
context, patients diagnosed with cancer preferred their physician to play a role in the decision making process because of his/her expertise. Nevertheless, most of the patients said that the illness trajectory would influence their preferred level of participation and that their preference might change along with the phase of illness [2].

\subsubsection{Investigate the Impact of Patient's Involvement in EOL Discussions}

The impact of ACP on EOL care was investigated in many studies [5] [6] [29]. Involvement in EOL discussions and the use of ACP increased patients' involvement in decision making or the appointment of surrogate decision makers, and improved the recognition of patients' wishes [6]. Those patients requested relieve from suffering, accepted the do-not-resuscitate order, received fewer aggressive medical interventions near death, and their deaths were associated with positive comments compared with deaths in patients who were not involved in EOL discussions [5]. Moreover, family members of patients who had died after being involved in EOL had fewer symptoms of posttraumatic stress, depression, anxiety, were prepared for patient's death and satisfied with its quality [5] [6].

One study examined the effectiveness of a nurse-led facilitated ACP intervention [29] and reported increasing the acceptance of ACP following discussion with a nurse facilitator. The same study revealed that ACP discussion with loved ones was associated with higher social support, while discussion with doctors was associated with lower quality of life. In fact, the number of facilitated ACP discussions with the nurse facilitator, and a preference for the ACP intervention were associated with higher acceptance of ACP [29].

\subsubsection{Barriers and Facilitators of EOL Discussions}

Barriers to initiate EOL discussions might be related to knowledge, attitude or behaviors. Knowledge related barriers are lack of awareness, lack of education and training in initiating and discussing difficult topics with patients [1] [20]; concerns about ethical and legal issues relating to withdrawing and withholding therapies; and the uncertainty of a disease trajectory [20]. On the other hand, attitude related barriers are fear of not being able to answer patients' questions or destroying hope; personal preferences of doctors or nurses affect discussions [20]; lack of experience and confidence with EOL discussions [1] [20].

Behaviors related barriers can be related to patient and family factors: reluctance of the family or patient to engage in discussions, a desire to protect the patient from "painful" information and the patient's lack of readiness or language barriers; or institutional factors: cultural barriers, a stigma around palliative care or lack of protocol, tools and training lack of time to develop a rapport with the patient or next of kin and limited resources [20]. Additionally, insufficient communication within the healthcare team and inaccurate or incomplete documentation were major challenges against EOL discussions.

In the same context, barriers to EOL discussions may arise from patients 
themselves or their relatives; not having felt sick enough, preferring to concentrate on staying alive, and not being sure which doctor would be providing care were the main reported patients' barriers [25], lack of adequate timing, and different patient and family expectations [20] were reported. On the other hand, patient's facilitators to EOL discussions were high levels of anxiety of upcoming future, fear of becoming a burden on relatives, and the experience of a beloved one death [25].

\section{Discussion}

\subsection{Summary of Evidence}

The aim of this review was to provide a comprehensive understanding of patients' involvement in decision making at EOL. Our systematic review identified eight review papers, six qualitative research studies, six observational studies and two clinical trials. Overall, there was diversity in studies that examined patient's involvement in the EOL decision making. The available literature described the current practices of patients' involvement, discussed perspectives of EOL discussions, investigated the impact and finally identified the barriers and facilitators of patients' involvement in EOL discussions.

Generally, there was a variation in current practices of patient involvement in the EOL decision making, while some patients requested to be involved [26], others wanted a natural death without interfering with GOD's arrangements [23], even a few number of patients actually documented their wishes in a written document or appointed a surrogate decision maker [14] [27]. These variations might be linked to the differences in cultural backgrounds of patients and their HCPs, many studies recommended to combine clinical information with values and beliefs of both patients and health care providers [13] [22] before planning for information disclosure. Current practice is also affected by the local policies of different hospitals; the availability of clear guidelines, along with assessing patient's willingness or readiness to be involved in EOL decisions will unify the practice dramatically facilitate their involvement.

On the other hand, involving nurses may improve communications in EOL discussions. According to Adams [32] registered nurses play a major role in EOL decision making, such as: facilitating communication between the patient and HCPs, providing support to patients and their families, and advocating for patients' rights. The use of a decisional coach into the process of care is another strategy to improve the decision making practices. The decisional coach would be someone (e.g., a nurse) who could understand patient-specific values and goals of care, and relay issues to physicians in preparation for the patient-physician encounter [33].

Level of education, health status and age were linked to patients' preferences of involving in EOL decision making [26]. Another variation in patients' preferences was found across different settings; while hospice care and nursing home residents make decisions in the context of the realization that one's life is near its 
end, cancer patients stated that illness trajectory would influence their preferred level of participation and their preference might change along with the phase of illness.

\subsection{Strengths and Limitations}

The strength of our study lies in its rigorous search strategies; the use of two separate researchers in assessing studies for screening, eligibility, and risk of bias, with secondary checking and verification of data extraction; as well as the use of PRISMA guideline to extensively examine the literature of almost 20 years that provided stronger evidence. Our review is limited by the highly heterogeneous nature of the research methodology, populations and measured outcomes. Other limitations include restricting to English-language publications and variations in quality of the included studies.

\subsection{Conclusions}

This paper provided an overview of the patients' involvement in the EOL decision making. Involvement in EOL discussions improved the recognition of patients' wishes, improved death experience, decreased posttraumatic stress, depression, and anxiety among family members. Despite the documented benefits, some barriers against patient's involvement in EOL decisions were recognized, lack of awareness; lack of education, training and experience; concerns about ethical and legal issues; and personal preferences of doctors or nurses were among the most common identified barriers.

Generally, there is a variation in the practices of the patient's involvement in EOL decisions and in the perspectives of patients, relatives and health care providers. This may guide future research to provide insights into the patient's preferences in order to improve the patient's experience of EOL care and decisions. Additionally, the literature highlighted the major barriers and facilitators of involving patients, which might help in improving the current practices. The limited studies within the Middle East culture demand the need for future studies to understand the impact of cultural context on patients' involvement and preferences.

\section{Key Statements}

\section{1) What is already known about the topic?}

- Patients differ in their preference for participation in treatment decisions at EOL.

- Patients' involvement in decisions at EOL improved the recognition of patients' wishes, improved death experience, decreased posttraumatic stress, depression, and anxiety among family members.

- Barriers against patient's involvement in EOL decisions are lack of awareness; lack of education, training and experience; concerns about ethical and legal issues; and personal preferences of doctors or nurses. 


\section{2) What this paper adds?}

- This paper has given an overview of the extent of patients' involvement in the EOL decision making during the last 19 years.

- This paper addressed the variation in practices of the patient's involvement in EOL decisions and in perspectives of patients, relatives and health care providers.

- Generally the involvement of patients and relatives in EOL decision making was limited, inappropriate timing of EOL care discussions was reported.

- A formal risk of bias assessment of all studies revealed a substantial variability in quality among quantitative studies. However, the qualitative studies appraised exhibited a high level of quality.

3) Implications for practice, theory or policy?

- The review identified the major barriers and facilitators of involving patients, which might help in improving the current practices.

- Future research to provide insights into the patient's preferences in order to improve the patient's experience of EOL care and decisions are needed.

- The limited studies within the Middle East culture demands the need for future studies to understand the impact of cultural context on patients' involvement and preferences.

\section{Authorship}

The three authors shared the process of systematic retrieval, assessment and analysis of the research studies, details of each contribution is stated in methods sections. Results and manuscript preparation: EO, IK, RZ.

\section{Data Management and Sharing}

All data generated or analyzed during this study are included in this published article.

\section{Funding}

This research received no specific grant from any funding agency in the public, commercial, or not-for-profit sectors.

\section{Acknowledgements}

The authors thank King Hussein Cancer Foundation and Center, and The University of Jordan/School of Nursing for library facilitation and assistance.

\section{Conflicts of Interest}

The author(s) declare(s) that there is no conflict of interest.

\section{References}

[1] Brooks, L.A., Manias, E. and Nicholson, P. (2017) Communication and Decision-Making about End-of-Life Care in the Intensive Care Unit. American Journal 
of Critical Care, 26, 336-341. https://doi.org/10.4037/ajcc2017774

[2] Brom, L., et al. (2014) Patients' Preferences for Participation in Treatment Decision-Making at the End of Life: Qualitative Interviews with Advanced Cancer Patients. PLoS ONE, 9, e100435. https://doi.org/10.1371/journal.pone.0100435

[3] Sinuff, T., et al. (2015) Improving EOL Communication and Decision Making: The Development of a Conceptual Framework and Quality Indicators. Journal of Pain and Symptom Management, 49, 1070-1080. https://doi.org/10.1016/j.jpainsymman.2014.12.007

[4] Bollig, G., Gjengedal, E. and Rosland, J.H. (2015) They Know!-Do They? A Qualitative Study of Residents and Relatives Views on Advance Care Planning, End-ofLife Care, and Decision-Making in Nursing Homes. Palliative Medicine, 30, 456-470. https://doi.org/10.1177/0269216315605753

[5] Wright, A.A., et al. (2008) Associations between EOL Discussions, Patient Mental Health, Medical Care near Death, and Caregiver Bereavement Adjustment. The Journal of the American Medical Association, 300, 1665-1673. https://doi.org/10.1001/jama.300.14.1665

[6] Detering, K.M., Hancock, A.D., Reade, M.C. and Silvester, W. (2010) The Impact of Advance Care Planning on End of Life Care in Elderly Patients: Randomised Controlled Trial. British Medical Journal, 340, c1345. https://doi.org/10.1136/bmj.c1345

[7] Teno, J.M., et al. (2004) Family Perspectives on EOL Care at the Last Place of Care. The Journal of the American Medical Association, 291, 88-93. https://doi.org/10.1001/jama.291.1.88

[8] Liberati, A., et al. (2009) The PRISMA Statement for Reporting Systematic Reviews and Meta-Analyses of Studies that Evaluate Health Care Interventions: Explanation and Elaboration. Journal of Clinical Epidemiology, 62, e1-e34.

https://doi.org/10.1016/j.jclinepi.2009.06.006

[9] Riva, J.J., Malik, K.M., Burnie, S.J., Endicott, A.R. and Busse, J.W. (2012) What Is Your Research Question? An Introduction to the PICOT Format for Clinicians. The Journal of the Canadian Chiropractic Association, 56, 167-171. https://www.ncbi.nlm.nih.gov/pmc/articles/PMC3430448/

[10] Effective Public Health Practice Project (1998) Quality Assessment Tool for Quantitative Studies Component Ratings a Selection Bias. 2-5. https://merst.ca/wp-content/uploads/2018/02/quality-assessment-tool 2010.pdf

[11] Critical Appraisal Skills Programme (2018) CASP (Qualitative) Checklist. https://casp-uk.net/casp-tools-checklists/

[12] Critical Appraisal Skills Programme (2018) CASP (Systematic Reviews) Checklist. https://casp-uk.net/casp-tools-checklists/

[13] Baggs, J.G. and Schmitt, M.H. (2000) EOL Decisions in Adult Intensive Care: Current Research Base and Directions for the Future. Nursing Outlook, 48, 158-164. https://doi.org/10.1067/mno.2000.100364

[14] Yadav, K.N., et al. (2017) Approximately One in Three Us Adults Completes Any Type of Advance Directive for EOL Care. Health Affairs, 36, 1244-1251. https://doi.org/10.1377/hlthaff.2017.0175

[15] Zwakman, M., et al. (2018) Advance Care Planning: A Systematic Review about Experiences of Patients with a Life-Threatening or Life-Limiting Illness. Palliative Medicine, 32, 1305-1321. https://doi.org/10.1177/0269216318784474

[16] Dening, K.H., Jones, L. and Sampson, E.L. (2011) Advance Care Planning for People with Dementia: A Review. International Psychogeriatrics, 23, 1535-1551. 
https://doi.org/10.1017/S1041610211001608

[17] Ke, L.S., Huang, X., Hu, W.Y., O'Connor, M. and Lee, S. (2017) Experiences and Perspectives of Older People Regarding Advance Care Planning: A Meta-Synthesis of Qualitative Studies. Palliative Medicine, 31, 394-405.

https://doi.org/10.1177/0269216316663507

[18] Mignani, V., Ingravallo, F., Mariani, E. and Chattat, R. (2017) Perspectives of Older People Living in Long-Term Care Facilities and of Their Family Members toward Advance Care Planning Discussions: A Systematic Review and Thematic Synthesis. Clinical Interventions in Aging, 12, 475-484. https://doi.org/10.2147/CIA.S128937

[19] Toro-Flores, R., López-González, R. and López-Muñoz, J.A. (2017) Knowledge and Attitudes of Critically Ill Patients and Their Families on Advance Directives and the Decision Making Process at the End of Life. Enfermería Intensiva, 28, 21-30. https://doi.org/10.1016/j.enfie.2016.07.001

[20] Travers, A. and Taylor, V. (2016) What Are the Barriers to Initiating EOL Conversations with Patients in the Last Year of Life? International Journal of Palliative Nursing, 22, 454-462. https://doi.org/10.12968/ijpn.2016.22.9.454

[21] Gauthier, D.M. and Swigart, V.A. (2003) The Contextual Nature of Decision Making Near the End of Life: Hospice Patients' Perspectives. American Journal of Hospice and Palliative Medicine, 20, 121-128. https://doi.org/10.1177/104990910302000210

[22] Gjerberg, E., Lillemoen, L., Forde, R. and Pedersen, R. (2015) EOL Care Communications and Shared Decision-Making in Norwegian Nursing Homes-Experiences and Perspectives of Patients and Relatives. BMC Geriatrics, 15, 103.

https://doi.org/10.1186/s12877-015-0096-y

[23] Ko, E. and Nelson-Becker, H. (2013) Does EOL Decision Making Matter? American Journal of Hospice and Palliative Medicine, 31, 183-188. https://doi.org/10.1177/1049909113482176

[24] Aldawood, A.S., et al. (2012) EOL Practices in a Tertiary Intensive Care Unit in Saudi Arabia. Anaesthesia and Intensive Care, 40, 137-141. https://doi.org/10.1177/0310057X1204000116

[25] Fakhri, S., et al. (2017) Factors Affecting Patients' Preferences for and Actual Discussions about End-of-Life Care. Journal of Pain and Symptom Management, 52, 386-394. https://doi.org/10.1016/j.jpainsymman.2016.03.012

[26] Levinson, W., Kao, A., Kuby, A. and Thisted, R.A. (2004) Not All Patients Want to Participate in Decision Making A National Study of Public Preferences. Journal of General Internal Medicine, 20, 531-535. https://doi.org/10.1111/j.1525-1497.2005.04101.x

[27] Waller, A., Turon, H., Bryant, J., Zucca, A., Evans, T.-J. and Sanson-Fisher, R. (2019) Medical Oncology Outpatients' Preferences and Experiences with Advanced Care Planning: A Cross-Sectional Study. BMC Cancer, 19, 63.

https://doi.org/10.1186/s12885-019-5272-6

[28] Young, K.A., Redfield, M.M., Strand, J.J. and Dunlay, S.M. (2017) EOL Discussions in Patients with Heart Failure. Journal of Cardiac Failure, 23, 821-825. https://doi.org/10.1016/j.cardfail.2017.08.451

[29] Sinclair, C., et al. (2017) Advance Care Planning Uptake among Patients with Severe Lung Disease: A Randomised Patient Preference Trial of a Nurse-Led, Facilitated Advance Care Planning Intervention. BMJ Open, 7, e013415.

https://doi.org/10.1136/bmjopen-2016-013415 
[30] Thomas, B.H., Ciliska, D., Dobbins, M. and Micucci, S. (2004) A Process for Systematically Reviewing the Literature: Providing the Research Evidence for Public Health Nursing Interventions. Worldviews on Evidence-Based Nursing, 1, 176-184. https://doi.org/10.1111/j.1524-475X.2004.04006.x

[31] Thomas, B.H., Ciliska, D., Dobbins, M. and Micucci, S. (1998) Dictionary for Quality Assessment Tool for Quantitative Studies (EPHPP).

https://merst.ca/wp-content/uploads/2018/02/qualilty-assessment-dictionary 2017. pdf

[32] Adams, J.A., Bailey, D.E., Anderson, R.A. and Docherty, S.L. (2011) Nursing Roles and Strategies in EOL Decision Making in Acute Care: A Systematic Review of the Literature. Nursing Research and Practice, 2011, Article ID: 527834. https://doi.org/10.1155/2011/527834

[33] Gainer, R.A., Curran, J., Buth, K.J., David, J.G., Légaré, J.F. and Hirsch, G.M. (2017) Toward Optimal Decision Making among Vulnerable Patients Referred for Cardiac Surgery: A Qualitative Analysis of Patient and Provider Perspectives. Medical Decision Making, 37, 600-610. https://doi.org/10.1177/0272989X16675338 


\section{Supplementary}

Table A1. Effective Public Health Practice Project (EPHPP) quality assessment tool for quantitative studies $(n=8)$.

\begin{tabular}{ccccccccccc}
\hline & & {$[24]$} & {$[6]$} & {$[25]$} & {$[26]$} & {$[29]$} & {$[27]$} & {$[5]$} & {$[28]$} \\
\hline 1 & Selection Bias & 1 & 1 & 3 & 2 & 1 & 1 & 3 & 2 \\
2 & Study Design & 3 & 1 & 3 & 3 & 1 & 3 & 3 & 3 \\
3 & Control for Confounders & 3 & 2 & 1 & 1 & 1 & 3 & 3 & 3 & 3 \\
4 & Blinding & 3 & 1 & 3 & 2 & 1 & 3 & 3 & 1 \\
5 & Data Collection Methods & 2 & 1 & 1 & 2 & 1 & 2 & 1 & 1 \\
6 & Withdrawals and Drop-Outs & 2 & 1 & 3 & 1 & 1 & 2 & 2 & 1 \\
\hline
\end{tabular}

1 = strong, 2 = moderate, 3 = weak.

Table A2. Critical Appraisals Skills Programme (CASP) checklist for included qualitative studies $(\mathrm{n}=6)$.

\begin{tabular}{|c|c|c|c|c|c|c|c|}
\hline & & {$[4]$} & [2] & {$[1]$} & [21] & [22] & [23] \\
\hline 1 & Was there a clear statement of the aims of the research? & 1 & 1 & 1 & 3 & 1 & 3 \\
\hline 2 & Is a qualitative methodology appropriate? & 1 & 1 & 1 & 1 & 1 & 1 \\
\hline 3 & Was the research design appropriate to address the aims of the research? & 1 & 1 & 1 & 1 & 1 & 1 \\
\hline 4 & Was the recruitment strategy appropriate to the aims of the research? & 1 & 1 & 1 & 1 & 1 & 1 \\
\hline 5 & Was the data collected in a way that addressed the research issue? & 1 & 1 & 1 & 1 & 1 & 1 \\
\hline 6 & Has the relationship between researcher and participants been adequately considered? & 1 & 3 & 3 & 3 & 2 & 3 \\
\hline 7 & Have ethical issues been taken into consideration? & 1 & 1 & 1 & 2 & 1 & 2 \\
\hline 8 & Was the data analysis sufficiently rigorous? & 1 & 1 & 1 & 1 & 1 & 1 \\
\hline 9 & Is there a clear statement of findings? & 1 & 1 & 1 & 1 & 1 & 1 \\
\hline
\end{tabular}

1 = yes, 2 = can't tell, 3 = no.

Table A3. Critical Appraisals Skills Programme (CASP) checklist for included systematic reviews $(\mathrm{n}=8)$.

\begin{tabular}{|c|c|c|c|c|c|c|c|c|c|}
\hline & & [13] & [16] & [17] & [18] & [19] & [20] & [14] & [15] \\
\hline 1 & Did the review address a clearly focused question? & 3 & 1 & 3 & 3 & 1 & 1 & 2 & 2 \\
\hline 2 & Did the authors look for the right type of papers? & 1 & 1 & 1 & 1 & 1 & 1 & 1 & 1 \\
\hline 3 & Do you think all the important, relevant studies were included? & 1 & 1 & 1 & 1 & 1 & 1 & 1 & 1 \\
\hline 4 & $\begin{array}{l}\text { Did the review's authors do enough to assess quality of the } \\
\text { included studies? }\end{array}$ & 3 & 2 & 1 & 1 & 1 & 1 & 2 & 1 \\
\hline 5 & $\begin{array}{l}\text { If the results of the review have been combined, was it reasonable } \\
\text { to do so? }\end{array}$ & 1 & 1 & 1 & 1 & 1 & 1 & 1 & 1 \\
\hline 6 & How precise are the results? & 1 & 1 & 2 & 1 & 1 & 1 & 1 & 1 \\
\hline 7 & Can the results be applied to the local population? & 1 & 1 & 1 & 1 & 1 & 1 & 1 & 1 \\
\hline 8 & Were all important outcomes considered? & 1 & 1 & 1 & 1 & 1 & 1 & 1 & 1 \\
\hline 9 & Are the benefits worth the harms and costs? & 1 & 1 & 1 & 1 & 1 & 1 & 1 & 1 \\
\hline
\end{tabular}

$1=$ yes, 2 = can't tell, 3 = no.

Table A4. https://mega.nz/\#!plYFgAIA!lj5szPLv3276v4Ha8Ww4xLHgdXBdDMif9LizUFPnO4o 